\title{
Serum prolidase levels and its relation with severity of acne vulgaris.
}

\author{
Elif Günay ${ }^{1}$, Selma Korkmaz ${ }^{2 *}$, Fatma Nesrin Turan ${ }^{3}$ \\ ${ }^{1}$ Department of Dermatology, Trakya University, Edirne, Turkey \\ ${ }^{2}$ Department of Dermatology, Süleyman Demirel University, Edirne, Turkey \\ ${ }^{3}$ Department of Biostatistics, Trakya University, Edirne, Turkey
}

\begin{abstract}
Background: The pathogenesis of acne vulgaris is multifactorial and inflammation plays a prominent role in the process. In clinical trials, independent from tissue type; it is found out that prolidase levels and activity are differing in numerous malign and chronic inflammatory processes on which collagen turnover is accelerated. This study aims to evaluate the relationship between serum prolidase levels and acne severity in acne vulgaris.

Methods: Thirty patients with mild to moderate Acne Vulgaris (AV), 30 with severe AV and 28 healthy volunteers were included in the study. The GAS was used to determine disease severity in patients with AV. Serum prolidase levels were measured by ELISA method.

Results: There were not statistically different between the groups in term of serum prolidase levels ( $p=0.819)$. In addition, there was no statistically significant difference between the two groups $(p=0.647$ for $\mathrm{AV}$ groups, $\mathrm{p}=\mathbf{0 . 9 2 6}$ for severe $\mathrm{AV}$ and healthy control, and $\mathrm{p}=\mathbf{0 . 5 3 9}$ for mild-moderate $\mathrm{AV}$ and healthy control). There was no statistically significant correlation between GAS and prolidase levels in patients with severe $A V(r=0.207, p=0.273)$ and mild-moderate $A V(r=0.162, p=0.392)$.

Conclusions: In conclusion, there was no significant difference in serum prolidase levels in patients with AV. New studies with wide case series are necessary to determine the role of prolidase on the pathogenesis of acne vulgaris.
\end{abstract}

Keywords: Acne, Inflammation, Prolidase.

Accepted on January 30, 2018

\section{Introduction}

Acne Vulgaris (AV) is an inflammatory disease of the pilosebaceous unit that is usually seen in adolescents and young adults. With the effects of androgens arising during puberty, sebum synthesis and secretion by the sebaceous glands accelerates. As a result, sebum accumulates in the follicular duct and destroys the follicle wall. After this damage, sebum, keratin, Propionibacterium acnes (P. acnes) and its enzymatic activity products (from the hydrolysis of sebum), and free fatty acids spread into the surrounding tissue. These immunogenic agents play a prominent role in the production of proinflammatory cytokines and initiating an inflammatory response in perifollicular tissue [1].

With the inflammation, angiogenesis and fibroblast migration occurs, and Matrix Metalloproteinases (MMP) are activated in the tissue. In the collagen cycle, prolidase, a cytosolic exopeptidase, plays a prominent role in the last step of the degradation of proline containing peptides and in recycling them. Prolidase is important in the rearrangement of growth and transcription factors, in wound healing, inflammation and angiogenesis [2].
Studies have determined that in dermatological conditions like psoriasis vulgaris and keloids, as well as in diseases with an accelerated collagen cycle like rheumatoid arthritis, and in chronic liver disease and osteoarthritis, Serum Prolidase Activity (SPA) is increased. Furthermore, serum prolidase, a sensitive inflammation marker, is found at increased levels in many chronic inflammatory diseases with accelerated collagen turnover [3]. Prolidase levels are also higher in the wound healing process [4]. To our knowledge, there is no study in the literature about inflammation and scar formation and their relationship to serum prolidase levels in the acne vulgaris.

This study aims to evaluate the relationship between serum prolidase levels and acne severity in acne vulgaris.

\section{Material and Method}

We conducted this study from March 2015 to August 2016 with patients from our dermatology clinic. We obtained permission from the local ethical committee as well as from our volunteers. The patients who were taking acne-inducing medications or who had a history of endocrinopathy, a 
malignancy or a systemic disease (an autoimmune disease or an infectious disease) were excluded.

Thirty patients with mild to moderate AV Global Acne Scoring System (GAS 1-30), 30 with severe AV (GAS $\geq 31$ ) and 28 healthy volunteers were included in the study. The GAS was used to determine disease severity in patients with AV [5]. Physical examinations of the participants were completed. Serum prolidase levels were measured via the Enzyme Linked Immunosorbent Assay (ELISA) method (Cusabio Biotech Co., Ltd., China).

\section{Statistical analysis}

Statistical evaluation was performed using the SPSS 22 statistical program. The normal distribution fitness of the measurable data was assessed by the Kolmogorov-Smirnov test, and then a t-test was performed in independent groups to measure intergroup differences for normal distributions. The Mann-Whitney $U$ test and the Kruskal-Wallis variance analysis were used to compare groups in evaluating data from nonnormal distributions. Spearman rho correlation analysis was performed for the relationship between the GAS scores and serum prolidase levels.

The Pearson chi-square test was used for qualitative data (gender). Median (min-max) values and the mean \pm the standard deviation were given as descriptive statistics. After the Kruskal-Wallis variance analysis, it was determined that $\mathrm{p}<0.017$ by making the Bonferroni correction for comparisons.

\section{Results}

There was no statistically significant difference based on gender between the groups of acne cases $(p=0.468)$. In the group with severe AV, the GAS mean was 33.30, while the mild to moderate AV group had a GAS mean of 14.23.

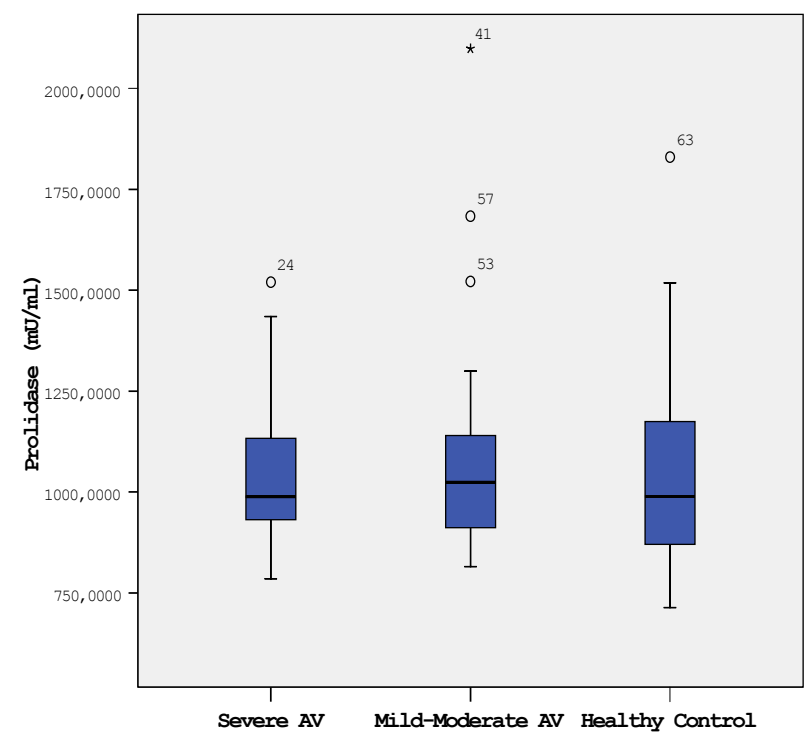

Figure 1. Prolidase levels of all groups.
Serum prolidase levels were 1,038.72 $( \pm$ 191.83) in severe AV; were $1,084.11( \pm 274.02)$ in mild to moderate $\mathrm{AV}$; and were $1,050.77( \pm 245.20)$ in the healthy control group (Figure 1). There were no statistically significant differences between the groups in terms of serum prolidase levels $(p=0.819)$. In addition, there were no statistically significant differences between the two groups in terms of prolidase level $(p=0.647$ for the AV groups, $\mathrm{p}=0.926$ for the severe AV group and healthy controls, and $\mathrm{p}=0.539$ for the mild to moderate $\mathrm{AV}$ group and healthy controls).

There was no statistically significant correlation between the GAS scores and prolidase levels in patients with severe AV $(\mathrm{r}=0.207, \mathrm{p}=0.273)$ and mild to moderate AV $(\mathrm{r}=0.162$, $\mathrm{p}=0.392$ ).

\section{Discussion}

In this study, there was no difference in prolidase levels between acne vulgaris patients and healthy controls.

Prolidase is acknowledged as the key enzyme for proline liberation and hydroxyproline residues on the collagen metabolism and cycle and letting them join to collagen structure. Prolidase activity levels vary with the destruction of collagen, which is found in different types and different ratios on the extracellular matrix [6-16].

There are many studies which indicate that prolidase levels are elevated in situations with scarring and chronic inflammation. It was found that prolidase levels are elevated by non-alcoholic steatohepatitis, and this elevation has a positive correlation with lobular inflammation and fibrosis $[13,16]$. Prolidase levels were found to be elevated in children having chronic hepatitis $\mathrm{B}$ infections, and this correlates with heaviness and the activity of the hepatitis B virus [17]. Correspondingly, another study found that prolidase levels rise in Helicobacter pylori infections [14].

Acne vulgaris is a chronic inflammatory disease that can result in scarring. It is acknowledged that increased inflammation and the severity of the acne aggravates the risk for developing cicatrices. It is known that scarring develops with an increase in acne severity. Acne and atrophic type cicatrices are most commonly seen in acne vulgaris [18]. Guven et al. [4] and Surucu et al. [19] have shown that, prolidase levels were found to be higher than those of the control groups, independent of the severity of the disease in chronic plaque psoriasis. In our study, in the group having mild to moderate $\mathrm{AV}$, serum prolidase levels were higher than in the severe AV and healthy control groups, but the results were not statistically significant $(p=0.539)$. This finding caused us to hypothesize that prolidase might be a factor related to developing inflammation; that it related to the initiation of inflammation in the microcomedonal stage. It also caused us to hypothesize that serum prolidase levels were decreasing due to collagen destruction in the process of scar formation.

Eren et al. [20] have shown that prolidase levels are correlated with high levels of C-Reactive Protein (CRP), and are higher in 
diabetic patients having foot ulcers than in diabetic patients without foot ulcers and in healthy controls. As a result of this study, they put forth the argument that prolidase activity could be a sensitive marker reflecting the inflammatory process.

The limitations of the study were the small numbers of participants in the groups, and the fact that no substantial inflammatory marker synchronous with prolidase was investigated.

In conclusion, there was no significant difference in serum prolidase levels in patients with AV. However, to fully evaluate the relationship between prolidase and $\mathrm{AV}$, molecular studies utilizing large groups of cases may be important. For this reason, new studies are needed in the future.

\section{Conflict of Interest}

The authors declare that they have no conflict of interest.

\section{References}

1. Lwin SM, Kimber I, Mc Fadden JP. Acne, quorum, sensing and danger. Clin Exp Dermatol 2014; 39: 162-167.

2. Erbagci AB, Araz M, Erbagci A, Tarakcioglu M, Namiduru ES. Serum prolidase activity as a marker of osteoporosis in type 2 diabetes mellitus. Clin Biochem 2002; 35: 263-268.

3. Ucar D, Em S, Bozkurt M, Oktayoglu P, Kurt Yüksel H, Çaglayan M. Serum prolidase activity in ankylosing spondylitis and rheumatoid arthritis. Clin Med Insights Arthritis Musculoskelet Disord 2013; 6: 29-33.

4. Guven B, Can M, Genc M, Koca R. Serum prolidase activity in psoriasis patients. Arch Dermatol Res 2013; 305 : 473-476.

5. Doshi A, Zaheer A, Stiller MJ. Acomparation of current acne grading systems and proposal of a novel system. Int $\mathrm{J}$ Dermatol 1997; 36: 416-418.

6. Senboshi Y, Oono T, Arata J. Localization of prolidase gene expression in scar tissue using in situ hybridization. J Dermatol Sci 1996; 12: 163-171.

7. Duong HS, Zhang QZ, Le AD, Kelly AP, Kamdar R, Messadi DV. Elevated prolidase activity in keloids: correlation with type I collagen turnover. Br J Dermatol 2006; 154: 820-828.

8. Karna E, Surazynski A, Palka JA. Collagen metabolism disturbances are accompanied by an increase in prolidase activity in lung carcinoma planoepitheliale. Int J Exp Pathol 2000; 81: 341-347.

9. Ilikhan SU, Bilici M, Sahin H, Akca ASD, Can M, Oz II. Assessment of the correlation between serum prolidase and alpha-fetoprotein levels in patients with hepatocellular carcinoma. World J Gastroenterol 2015; 21: 6999-7007.
10. Pasko-Cechowska M, Palka J, Wojtukiewicz M Z. Enhanced prolidase activity and decreased collagen content in breast cancer tissue. Int J Exp Pathol 2006; 87: 289-296.

11. Gecit I, Aslan M, Gunes M, Pirincci N, Esen R, Demir H. Serum prolidase activity, oxidative stress, and nitric oxide levels in patients with bladder cancer. J Cancer Res Clin Oncol 2012; 138: 739-743.

12. Arioz DT, Camuzcuoglu H, Toy H, Kurt S, Celik H, Aksoy N. Serum prolidase activity and oxidative status in patients with stage I endometrial cancer. Int J Gynecol Cancer 2009; 19: 1244-1247.

13. Kayadibi H, Gultepe M, Yasar B, Ince AT, Ozcan O, Ipcioglu OM, Kurdas OO. Diagnostic value of serum prolidase enzyme activity to predict the liver histological lesions in non-alcoholic fatty liver disease: a surrogate marker to distinguish steatohepatitis from simple steatosis. Dig Dis Sci 2009; 54: 1764-1771.

14. Aslan M, Nazligul Y, Horoz M, Bolukbas C, Bolukbas FF, Aksoy N. Serum prolidase activity and oxidative status in Helicobacter pylori infection. Clin Biochem 2007; 40: 37-40.

15. Savas E, Aksoy N, Pehlivan Y, Sayiner ZA, Öztürk ZA, Tabur S. Evaluation of oxidant and antioxidant status and relation with prolidase in systemic sclerosis. Wien Klin Wochenschr 2014; 126: 341-346.

16. Horoz M, Aslan M, Bolukbas FF, Bolukbas C, Nazligul Y, Celik H. Serum prolidase enzyme activity and its relation to histopathological findings in patients with non-alcoholic steatohepatitis. J Clin Lab Anal 2010; 24: 207-211.

17. Şen V, Uluca U, Ece A, Kaplan I, Bozkurt F, Aktar F. Serum prolidase activity and oxidant-antioxidant status in children with chronic hepatitis B virus infection. Ital J Pediatr 2014; 40: 95.

18. Fabbrocini G, Annunziata MC, D Arco V, De Vita V, Lodi G, Mauriello MC. Acne scars: pathogenesis, classification and treatment. Dermatol Res Pract 2010; 2010: 893080.

19. Surucu HA, Aksoy N, Ozgoztas O, Sezen H, Yesilova Y, Turan E. Prolidase activity in chronic plaque psoriasis patients. Postepy Dermatol Alergol 2015; 32: 82-87.

20. Eren MA, Torun AN, Tabur S, Ulas T, Demir M, Sabuncu T. Serum prolidase activity in diabetic foot ulcers. Acta Diabetol 2013; 50: 423-427.

\section{*Correspondence to}

\section{Selma Korkmaz}

Department of Dermatology

Süleyman Demirel University

Turkey 